\title{
COMPARING THE RELATIVE VOLUME WITH THE RELATIVE INRADIUS AND THE RELATIVE WIDTH
}

\author{
A. CERDÁN \\ Received 28 February 2006; Revised 24 August 2006; Accepted 29 August 2006
}

We consider subdivisions of a convex body $G$ in two subsets $E$ and $G \backslash E$. We obtain several inequalities comparing the relative volume: (1) with the minimum relative inradius, (2) with the maximum relative inradius, (3) with the minimum relative width, and (4) with the maximum relative width. In each case, we obtain the best upper and lower estimates for subdivisions determined by general hypersurfaces and by hyperplanes.

Copyright ( 2006 A. Cerdán. This is an open access article distributed under the Creative Commons Attribution License, which permits unrestricted use, distribution, and reproduction in any medium, provided the original work is properly cited.

\section{Introduction}

Let $G$ be an open bounded convex set. Let us consider subdivisions of $G$ in two connected subsets $E$ and $G \backslash E$ in such a way that the relative boundary $(\partial E \cap G)$ is a connected topological hypersurface.

Relative geometric inequalities compare functionals that give information about the geometry of the subdivision; we call these functionals relative geometric measures.

This kind of inequalities provides either lower or upper estimates of the ratio between appropriate powers of two of those relative geometric measures. In case that these upper (or lower) estimates exist, we call the sets for which the equality sign is attained maximizers (or minimizers).

The oldest relative geometric inequalities that were investigated are the so-called relative isoperimetric inequalities. They provide upper estimates of the ratio between appropriate powers of the relative volume (the minimum between the volume of $E$ and the volume of its complement) and the relative perimeter (the length of the relative boundary). Many results about relative isoperimetric inequalities are included in $[3,5]$.

Recently some results have been obtained by comparing the relative perimeter and the minimum relative diameter (the minimum between the diameter of $E$ and the diameter of its complement) for subdivisions of planar convex sets (see [2]), and the relative volume with the maximal and the minimal relative diameters (see [1]). 
The aim of this paper is to study relative geometric inequalities concerning the relative volume, the relative inradius, and the relative width of a subset of a convex body. We define these notions in the following way.

Let $G$ be an open bounded convex set in $\mathbb{R}^{n}$ and $E \subset G$ a subset of $G$ such that $E$ as well as $G \backslash E$ are connected, have nonempty interior, and their relative boundary is a connected topological hypersurface.

The inradius $\rho(K)$ of a convex body $K$ is the greatest value for which there is a ball $B$ with radius $\rho(K)$ contained in $K$.

The minimal width $\omega(K)$ of a convex body $K$ is the shortest distance between pairs of parallel supporting hyperplanes.

(i) The relative volume is the minimum between the volume of $E$ and the volume of its complement

$$
V(E, G)=\min \{V(E), V(G \backslash E)\} .
$$

(ii) The minimum relative inradius is the minimum between the inradius of $E$ and the inradius of its complement

$$
\rho_{m}(E, G)=\min \{\rho(E), \rho(G \backslash E)\} .
$$

(iii) The maximum relative inradius is the maximum between the inradius of $E$ and the inradius of its complement

$$
\rho_{M}(E, G)=\max \{\rho(E), \rho(G \backslash E)\} .
$$

(iv) The minimum relative width is the minimum between the width of $E$ and the width of its complement

$$
\omega_{m}(E, G)=\min \{\omega(E), \omega(G \backslash E)\} .
$$

(v) The maximum relative width is the maximum between the width of $E$ and the width of its complement

$$
\omega_{M}(E, G)=\max \{\omega(E), \omega(G \backslash E)\} .
$$

We are going to obtain global upper and lower estimates of the ratios

$$
\frac{V(E, G)}{\rho_{m}(E, G)^{n}}, \quad \frac{V(E, G)}{\rho_{M}(E, G)^{n}}, \quad \frac{V(E, G)}{\omega_{m}(E, G)^{n}}, \quad \frac{V(E, G)}{\omega_{M}(E, G)^{n}} .
$$

We consider divisions by general hypersurfaces, but due to their interest - in the cases in which the estimates are different-we also consider divisions by hyperplanes.

\section{Relative geometric inequalities concerning the relative volume and the minimum relative inradius of a subset of an open bounded convex set}

The aim of this section is maximizing and minimizing the ratio between the relative volume and the $n$ th-power of the minimum relative inradius of a subset of an open bounded convex set. 


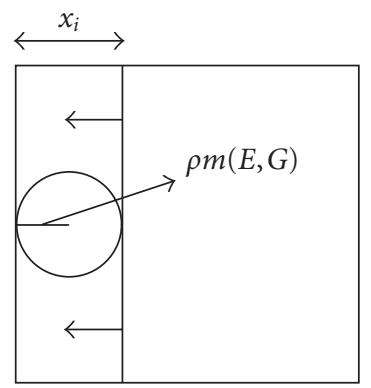

Figure 2.1

Proposition 2.1. Let $G$ be an open bounded convex set in $\mathbb{R}^{n}$ and $E$ a subset of $G$ such that $E$ and $G \backslash E$ are connected. Then there is no upper bound for the ratio

$$
\frac{V(E, G)}{\rho_{m}(E, G)^{n}}
$$

Proof. Let $G$ be the $n$-dimensional unit hypercube. We consider a sequence $\left\{\Pi_{i}\right\}$ of hyperplanes parallel to one of the facets, where $x_{i}=1 / i$ is the distance from $\Pi_{i}$ to the nearest facet of $G$ parallel to it, $i \geq 2$ (Figure 2.1).

Then,

$$
\lim _{i \rightarrow \infty} \frac{V\left(E_{i}, G\right)}{\rho_{m}\left(E_{i}, G\right)^{n}}=\lim _{i \rightarrow \infty} \frac{x_{i}}{\left(x_{i} / 2\right)^{n}}=\infty .
$$

Proposition 2.2. Let $G$ be an open bounded convex set in $\mathbb{R}^{n}$ and $E$ a subset of $G$ obtained by dividing $G$ by a general hypersurface such that $E$ and $G \backslash E$ are connected. Then,

$$
\frac{V(E, G)}{\rho_{m}(E, G)^{n}} \geq \frac{\pi^{n / 2}}{\Gamma(n / 2+1)} .
$$

The equality is attained when the hypersurface is the boundary of the inball.

Proof. Let $G$ be an open bounded convex set and let $E$ be a subset of $G$ obtained by dividing $G$ by a general hypersurface. If we consider the inball $B$ of $E$, this ball has the same inradius as $E$ and less volume, so

$$
\frac{V(E, G)}{\rho_{m}(E, G)^{n}} \geq \frac{V(B, G)}{\rho_{m}(B, G)^{n}}=\frac{\pi^{n / 2}}{\Gamma(n / 2+1)} .
$$

Proposition 2.3. Let $G$ be an open bounded convex set in $\mathbb{R}^{n}$ and $E$ a subset of $G$ obtained by a hyperplane cut. Then,

$$
\frac{V(E, G)}{\rho_{m}(E, G)^{n}} \geq \frac{\pi^{n / 2}}{2 \Gamma(n / 2+1)}+\frac{\pi^{(n-1) / 2}}{\Gamma((n-1) / 2+1)},
$$

where the equality is attained, for instance, for the optimal set described in Figure 2.2. 


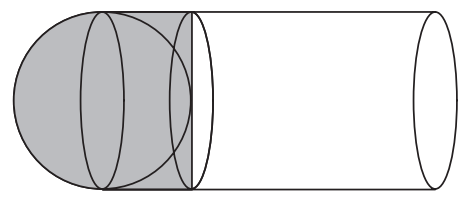

Figure 2.2

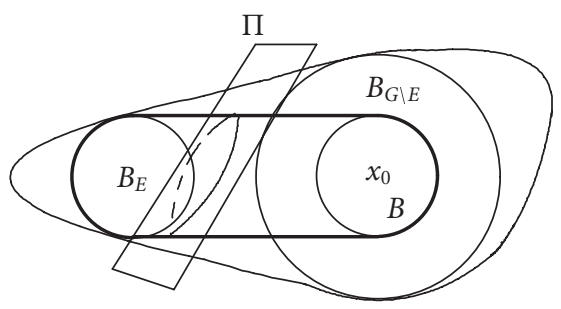

Figure 2.3

Proof. (1) Suppose that $\rho_{m}(E, G)=\rho(E)$ and $V(E, G)=V(E)$.

Let $E$ be a subset of $G$ obtained by dividing $G$ with a hyperplane $\Pi$ such that $\rho_{m}(E, G)=$ $\rho(E)$. Let $B_{E}$ be an inball of $E$ and let $B_{G \backslash E}$ be an inball of $G \backslash E$. Let $x_{0}$ be the center of $B_{G \backslash E}$. Let $B$ be a ball with center $x_{0}$ and radius $\rho(E)$. Then $B \subset B_{G \backslash E}$. We consider $C$ the convex hull of $B_{E} \cup B$. By the convexity of $G, C \subset G$. Then $\Pi$ divides $C$ into two subsets $E^{\prime}$ and $C \backslash E^{\prime}$ such that $\rho_{m}\left(E^{\prime}, C\right)=\rho_{m}(E, G)$ and $V\left(E^{\prime}, C\right)=V\left(E^{\prime}\right) \leq V(E, G)$ (Figure 2.3). So,

$$
\frac{V(E, G)}{\rho_{m}(E, G)^{n}} \geq \frac{V\left(E^{\prime}, C\right)}{\rho_{m}\left(E^{\prime}, C\right)^{n}} .
$$

The boundary of $C$ consists of two half spheres and a piece of cylinder. Let $\Pi^{\prime}$ be a hyperplane tangent to $B_{E}$ and spanned by all the normal vectors to the cylindrical hypersurface which bounds $C$. Then $\Pi^{\prime}$ divides $C$ into two subsets $E^{\prime \prime}$ and $C \backslash E^{\prime \prime}$ both of them with the same inradius $\rho_{m}(E, G)$, and such that $V\left(E^{\prime \prime}, C\right) \leq V\left(E^{\prime}, C\right)$ (Figure 2.4). So,

$$
\frac{V\left(E^{\prime}, C\right)}{\rho_{m}\left(E^{\prime}, C\right)^{n}} \geq \frac{V\left(E^{\prime \prime}, C\right)}{\rho_{m}\left(E^{\prime \prime}, C\right)^{n}}=\frac{\pi^{n / 2}}{2 \Gamma(n / 2+1)}+\frac{\pi^{(n-1) / 2}}{\Gamma((n-1) / 2+1)} .
$$

The example in Figure 2.2 shows that this is the best possible lower bound.

(2) Suppose that $\rho_{m}(E, G)=\rho(E)$ and $V(E, G)=V(G \backslash E)$.

In this case we can translate the hyperplane $\Pi$ obtaining a new subset $E^{\prime}$ such that $V\left(E^{\prime}, G\right)=V\left(G \backslash E^{\prime}\right) \leq V(G \backslash E)=V(E, G)$ and $\rho_{m}\left(E^{\prime}, G\right)=\rho\left(E^{\prime}\right)=\rho\left(G \backslash E^{\prime}\right) \geq \rho(E)=$ $\rho_{m}(E, G)$ (Figure 2.5). Then we stay in the previous case and so,

$$
\frac{V(E, G)}{\rho_{m}(E, G)^{n}} \geq \frac{V\left(E^{\prime}, G\right)}{\rho_{m}\left(E^{\prime}, G\right)^{n}} \geq \frac{\pi^{n / 2}}{2 \Gamma(n / 2+1)}+\frac{\pi^{(n-1) / 2}}{\Gamma((n-1) / 2+1)} .
$$




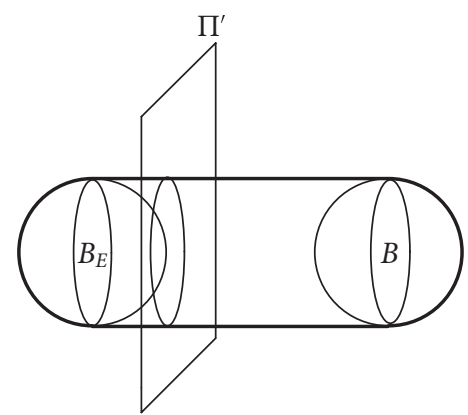

Figure 2.4

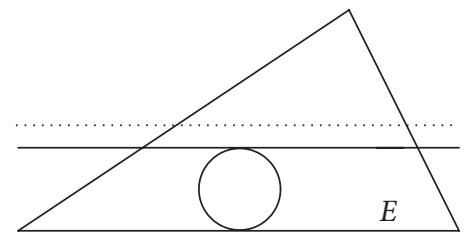

Figure 2.5

\section{Relative geometric inequalities concerning the relative volume and the maximum relative inradius of a subset of an open bounded convex set}

Now we are going to study the problem of maximizing and minimizing the ratio between the relative volume and the $n$ th-power of the maximum relative inradius of a subset of an open bounded convex set.

Proposition 3.1. Let $G$ be an open bounded convex set in $\mathbb{R}^{n}$ and $E$ a subset of $G$ such that $E$ and $G \backslash E$ are connected. Then there is no upper bound for the ratio

$$
\frac{V(E, G)}{\rho_{M}(E, G)^{n}}
$$

Proof. Let $G$ be a box in $\mathbb{R}^{n}$ with edge lengths $x_{1}, x_{2}, \ldots, x_{n}$, such that $x_{1}=\cdots=x_{n-1}, x_{n} \geq$ $x_{1} / 2$. We consider a division into two subsets of equal volume by a hyperplane parallel to a facet which contains the edge of length $x_{n}$.

Then,

$$
\frac{V(E, G)}{\rho_{M}(E, G)^{n}}=\frac{\left(x_{1}\right)^{n-1} x_{n} / 2}{\left(x_{1} / 4\right)^{n}}=\frac{4^{n} x_{n}}{2 x_{1}} .
$$

If we fix $x_{1}$, when $x_{n} \rightarrow \infty$, we have

$$
\lim _{x_{n} \rightarrow \infty} \frac{V(E, G)}{\rho_{M}(E, G)^{n}}=\lim _{x_{n} \rightarrow \infty} \frac{4^{n} x_{n}}{2 x_{1}}=\infty .
$$




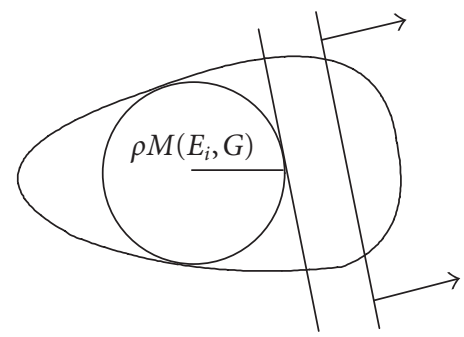

Figure 3.1

Proposition 3.2. Let $G$ be an open bounded convex set in $\mathbb{R}^{n}$ and $E$ a subset of $G$ such that $E$ and $G \backslash E$ are connected. Then, the ratio $V(E, G) / \rho_{M}(E, G)^{n}$ is obviously nonnegative and it is possible to find subdivisions of $G$ for which this ratio is as small as required.

Proof. We consider a sequence $\left\{\Pi_{i}\right\}$ of hyperplanes intersecting $G$ which determines a sequence $\left\{E_{i}\right\}$ of subsets of $G$ such that $V\left(E_{i}, G\right) \rightarrow 0$ and $\rho_{M}\left(E_{i}, G\right) \rightarrow \rho(G)$ when $i \rightarrow \infty$ (Figure 3.1). Then,

$$
\lim _{i \rightarrow \infty} \frac{V\left(E_{i}, G\right)}{\rho_{M}\left(E_{i}, G\right)^{n}}=0 .
$$

\section{Relative geometric inequalities concerning the relative volume and the minimum relative width of a subset of an open bounded convex set}

In this section we replace the inradius by another geometric functional, the minimal width, which for the sake of simplicity we will call the width, and we are going to compare the ratio between the relative volume and the $n$ th-power of the minimum relative width of a subset of an open bounded convex set.

Proposition 4.1. Let $G$ be an open bounded convex set in $\mathbb{R}^{n}$ and $E$ a subset of $G$ such that $E$ and $G \backslash E$ are connected. Then there is no upper bound for the ratio

$$
\frac{V(E, G)}{\omega_{m}(E, G)^{n}}
$$

The proof of this result follows from Proposition 2.1 (the counterexample considered in the proof is a centrally symmetric convex body so that the relative width equals twice the relative inradius).

Proposition 4.2. Let $G$ be an open bounded convex set in $\mathbb{R}^{n}$ and $E$ a subset of $G$ such that $E$ and $G \backslash E$ are connected. Then one can always find subsets $E$ of $G$ for which the ratio $V(E, G) / \omega_{m}(E, G)^{n}$ is as small as it is required.

Proof. Without loss of generality we can suppose that $0 \in G$. Let $\left\{E_{i}\right\}_{i \in \mathbb{N}}$ be a sequence of subsets of $G$ such that $E_{i}=(1-1 / i) G$, where $i \geq 2$ (Figure 4.1). 


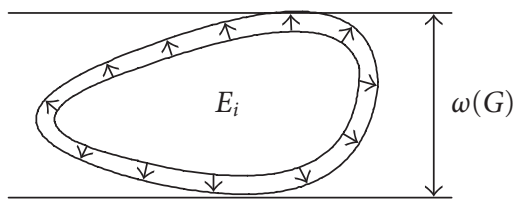

Figure 4.1

Then, for $i$ sufficiently large, $V\left(E_{i}, G\right)=V\left(G \backslash E_{i}\right)$ and $\omega_{m}\left(E_{i}, G\right)=\omega\left(E_{i}\right)$. So, when $i \rightarrow \infty$ we have that $V\left(E_{i}, G\right) \rightarrow 0$ and $\omega_{m}\left(E_{i}, G\right) \rightarrow \omega(G)$. Hence,

$$
\lim _{i \rightarrow \infty} \frac{V\left(E_{i}, G\right)}{\omega_{m}\left(E_{i}, G\right)^{n}}=\frac{0}{\omega(G)^{n}}=0
$$

To prove the next proposition we need the following result.

Theorem 4.3 (see [4]). Let $G$ be a bounded planar convex set and let $A(G)$ be the area of $G$, then,

$$
A(G) \geq \frac{\omega(G)^{2}}{\sqrt{3}}
$$

where the equality sign is attained if $G$ is an equilateral triangle.

Proposition 4.4. Let $G$ be an open bounded convex set in $\mathbb{R}^{2}$ and $E$ a subset of $G$ obtained by dividing $G$ with a straight line segment. Then,

$$
\frac{A(E, G)}{\omega_{m}(E, G)^{2}} \geq \frac{1}{\sqrt{3}}
$$

Proof. We first assume that $A(E, G)=A(E)$ and $\omega_{m}(E, G)=\omega(E)$. Then, as the subset $E$ is a bounded convex set, applying Theorem 4.3 , we obtain

$$
A(E) \geq \frac{\omega(E)^{2}}{\sqrt{3}} \quad \text { or equivalently } \quad \frac{A(E)}{\omega(E)^{2}} \geq \frac{1}{\sqrt{3}}
$$

Hence,

$$
\frac{A(E, G)}{\omega_{m}(E, G)^{2}}=\frac{A(E)}{\omega(E)^{2}} \geq \frac{1}{\sqrt{3}}
$$

If $A(E, G)=A(E)$ and $\omega_{m}(E, G)=\omega(G \backslash E)$, we translate the straight line segment that divides $G$ towards the half-plane containing $E$ until we obtain a new subdivision $\left\{E^{\prime}, G \backslash E^{\prime}\right\}$ such that $A\left(E^{\prime}, G\right)=A\left(E^{\prime}\right) \leq A(E)$ and $\omega_{m}\left(E^{\prime}, G\right)=\omega\left(E^{\prime}\right) \geq \omega(G \backslash E)$.

This proof cannot be extended to higher dimensions because Pal's problem is still open for $n \geq 3$. 
8 Comparing some relative geometric measures

\section{Relative geometric inequalities concerning the relative volume and the maximum} relative width of a subset of an open bounded convex set

Finally, we will maximize and minimize the ratio between the relative volume and the $n$ th-power of the maximum relative width of a subset of an open bounded convex set.

Using the same arguments as in Propositions 3.1 and 3.2, we can state the following.

Proposition 5.1. Let $G$ be an open bounded convex set in $\mathbb{R}^{n}$ and $E$ a subset of $G$ such that $E$ and $G \backslash E$ are connected. Then there is no upper bound for the ratio

$$
\frac{V(E, G)}{\omega_{M}(E, G)^{n}}
$$

Proposition 5.2. Let $G$ be an open bounded convex set in $\mathbb{R}^{n}$ and $E$ a subset of $G$ such that $E$ and $G \backslash E$ are connected. Then the ratio $V(E, G) / \omega_{M}(E, G)^{n}$ is obviously nonnegative and it is possible to find subdivisions of $G$ for which this ratio is as small as required.

\section{References}

[1] A. Cerdán, C. Miori, and S. Segura Gomis, Relative isodiametric inequalities, Beiträge zur Algebra und Geometrie 45 (2004), no. 2, 595-605.

[2] A. Cerdán, U. Schnell, and S. Segura Gomis, On relative geometric inequalities, Mathematical Inequalities \& Applications 7 (2004), no. 1, 135-148.

[3] A. Cianchi, On relative isoperimetric inequalities in the plane, Bollettino della Unione Matemàtica Italiana. Serie VII. B 3 (1989), no. 2, 289-325.

[4] J. Pál, Ein Minimumproblem für Ovale, Mathematische Annalen 83 (1921), no. 3-4, 311-319.

[5] C. Peri, On relative isoperimetric inequalities, Conferenze del Seminario di Matematica dell’Università di Bari (2001), no. 279, 14.

A. Cerdán: Departamento de Análisis Matemático, Universidad de Alicante,

Campus de San Vicente del Raspeig, 03080 Alicante, Spain

E-mail address: aacs@alu.ua.es 